\title{
Branching scenarios in eco-evolutionary prey-predator models
}

Pietro Landi

Fabio Dercole

Sergio Rinaldi (rinaldi@iiasa.ac.at)

\section{Approved by}

Ulf Dieckmann

Director, Evolution and Ecology Program

June 2015

Interim Reports on work of the International Institute for Applied Systems Analysis receive only limited review. Views or opinions expressed herein do not necessarily represent those of the Institute, its National Member Organizations, or other organizations supporting the work. 


\title{
BRANCHING SCENARIOS IN ECO-EVOLUTIONARY PREY-PREDATOR MODELS
}

\author{
PIETRO LANDI*, FABIO DERCOLE ${ }^{\dagger}$, AND SERGIO RINALDI $\ddagger$
}

\begin{abstract}
We show in this paper how simulations of ODEs and continuations of systems of algebraic equations can be combined to study the evolution of biodiversity in multi-species systems where phenotypic traits are genetically transmitted. We follow the Adaptive Dynamics (AD) approach, that provides a deterministic approximation of the evolutionary dynamics of stationary coexisting populations in terms of an ODE system, the so-called AD canonical equation. AD also provides explicit conditions to test whether a stable evolutionary equilibrium of the canonical equation is a branching point-resident and mutant morphs coexist and further differentiate thus increasing biodiversity — or not. We analyze a standard parameterized family of prey-predator communities, described by the most standard ecological model, and propose an iterative procedure to obtain the branching portrait, explaining the dependence of branching scenarios on two (demographic, environmental or control) parameters. Among a number of interesting results, in line with field studies and known ecological principles, we find that prey branching is induced by the predation pressure, and is favored when prey intraspecific competition is highly sensitive to the resident-mutant phenotypic mismatch; while predator branching is not possible when prey and predators are present in equal number of morphs. This results in alternate prey-predator branching sequences with possible phases of prey unilateral branching. The guidelines for deriving a general method for analyzing the evolution of biodiversity are also discussed. The indications that can be obtained typically have qualitative nature, but can be of help for the long-term conservation and management of biodiversity.
\end{abstract}

Key words. Adaptive Dynamics, biodiversity, coevolution, evolutionary branching, polymorphism, prey-predator model

AMS subject classifications. 92D15, 34C23, 65L07

Introduction. Explaining why there are so many similar populations in Nature is one of the major questions in ecology [34]. Classical competition theory can provide some answers; for example, it can explain why self-organized patterns of groups of similar populations emerge in rich communities [54]. Perhaps the most difficult problem in need of explanation is this: under the assumption that life started from a common ancestor, how is it that we obverse so much biodiversity today? For this, we can combine two independent mechanisms. One is genetic mutation that rarely and randomly diversifies the phenotypic trait $(x)$ of some individual from that $\left(x^{\prime}\right)$ of its offspring, thus creating a mutant population similar to the resident one, though initially with very low abundance. The second is natural selection, i.e., the competition between resident and mutant populations, that generically leads to the extinction of one of the two populations [23, 13]. If mutants go extinct, nothing changes because $x$ remains the resident trait, while if residents go extinct, the mutant population becomes the new resident, endowed, however, of a new trait $x^{\prime}$. Only in exceptional cases, called evolutionary branchings [27, 25, 13], can the mutant and resident populations coexist under disruptive selection, i.e., competition favors further differentiation between the two similar residents. Evolutionary branching thus explains why the number of distinct morphs of a species, that is, biodiversity, can increase. Biodiversity can also decrease, not only accidentally, but as the result of evolution toward the boundary of the viability region in trait space (evolutionary extinction, see [30, 13]). Evolutionary branchings and extinctions can also alternate [20, 39, 6, 13], making the problem more complex.

*Dipartimento di Elettronica, Informazione e Bioingegneria, Politecnico di Milano, Via Ponzio 34/5, 20133 Milano, Italy Ph: +39 022399 4029; Fax: +39 022399 3412; email: landi@elet.polimi.it

${ }^{\dagger}$ Dipartimento di Elettronica, Informazione e Bioingegneria, Politecnico di Milano, Via Ponzio 34/5, 20133 Milano, Italy

${ }_{\ddagger}^{\ddagger}$ Dipartimento di Elettronica, Informazione e Bioingegneria, Politecnico di Milano, Via Ponzio 34/5, 20133 Milano, Italy, and Evolution and Ecology Program, International Institute for Applied Systems Analysis, Laxenburg, Austria 
Among the many quantitative approaches to evolutionary dynamics (seven are described in [13]) only two of them, that is, Individual-Based Modeling (IBM) and Adaptive Dynamics (AD) are suitable for predicting branching scenarios. IBM [5, 15] is a stochastic simulation approach which requires little in the way of mathematical analysis and skill, but which can be quite attractive because it allows any sort of detail to be included in the model (e.g. age, size, stage and spatial structures, sexual reproduction, and seasonalities). In contrast, AD $[49,27,25,13,24]$ is population-based, focused on the long-term evolution of the adaptive traits (it assumes rare mutations of small phenotypic effect), and provides a deterministic approximation of the evolutionary dynamics in terms of ODEs, one for each trait, known as the $\mathrm{AD}$ canonical equation [16, 4, 13]. Evolutionary branching is possible only in the vicinity of evolutionary equilibria (far from equilibria the extinction of either the resident or the mutant population is the generic outcome of competition [23, 13]), and explicit branching conditions are available [27, 25, 13]. Here we consider the simplest setting in which the $\mathrm{AD}$ canonical equation can be derived, i.e., the case of unstructured, asexual populations, characterized by a single trait each, and stationary coexisting in an isolated homogeneous and constant abiotic environment (see [16, 21, 38] for extensions).

Most branching scenarios produced until now have been obtained either analytically (for relatively simple models or limited to primary branchings, see e.g. [49, 25, 37, 20, 22, 7, 2]) or by means of stochastic simulations (with the IBM approach [15, 20, 22] or stochastically simulating the mutation process of an $\mathrm{AD}$ model [49, 25, 37, 2]), starting from an ancestral community composed of a single population or two populations of different species. In some studies concerning prey-predator systems the scenarios are extremely simple, ranging from no branching due to periodic evolutionary attractors in [17], to a primary prey branching followed by predator branching in [20] (a primary predator branching has been identified in [26], but relying on cyclically coexisting predators). By contrast, in studies on the coevolution of mutualism, richer scenarios characterized by alternate branching and extinction (in facultative or opportunistic mutualisms [20]) and by very long series of evolutionary branchings (in obligate mutualisms [22]) have been discovered. In [22] branching is unilateral in some regions of parameter space, that is, it concerns always populations of the same species, while in other regions it is bilateral and alternate between the two species. Alternate branchings have also been obtained in a study of a host-parasite model [2], while bilateral but not alternate branchings have been discovered in a study of evolution of cannibalistic populations toward complex food webs [35].

The above mentioned studies show that branching scenarios depend not only on the ancestral community but also on the parameter values characterizing some demographic processes (like efficiency or death rate) or their dependence on evolving traits. To find out the full catalog of branching scenarios, say in a two-dimensional parameter space, one cannot rely on simulation approaches, as this would be computationally impracticable. Instead, one could use the AD canonical equation and the branching conditions to generate a point on each curve separating regions of parameter space with different branching scenarios, and, then, produce the entire curve through numerical continuation techniques [1]. Until now, this has been done only a few times up to the first branching (in a study of seed size and competitive ability [28], in a study of prey-predator coevolution [11], in a model for the evolution of cannibalism [12, 6], and in modeling the host-parasite range [2]).

The aim of this paper is to show how the analysis of evolutionary branching can be organized through continuation in a case of complex scenarios. For this, we study the coevolution of a resource-consumer system starting from an ancestral prey-predator pair, the building block of complex food webs [46]. The model we consider is based on the preypredator model most frequently used in ecology, for which all properties, except branching 
scenarios, have already been studied in detail (the primary prey branching has been studied in [11], while the primary branching of cycling predators in [26]). This choice allows this paper to be considered as the natural follow-up of the work in [11] that was published in this journal. We feel our results are of interest because they both support ecological properties that emerge from field studies and suggest new theoretical insights on prey-predator coevolution that might be of help for the conservation and management of biodiversity. In the discussion, we also describe how our approach can be made general and applied to study the evolution of biodiversity in different communities.

Coevolution of prey-predator systems. Evolving systems are in general composed of $N$ interacting plant and/or animal populations characterizable by two features: the number $n_{i}$ of individuals of each population or, equivalently, the density of the population (a positive real number), and an adaptive phenotypic trait $x_{i}$ (e.g., body size, typically measured by a real number on a suitable scale [11]). Both features vary in time, but densities can vary at a much faster rate than traits. This means that an evolving system has two distinct timescales called ecological and evolutionary. The first is fast and concerns only the densities, while traits remain practically constant if mutations are assumed to be extremely rare events on the ecological timescale; the second concerns the slow variation of the traits, due to sequences of mutations and resident substitutions, and entrains the slow variations of the equilibrium densities attained after each substitution.

From now on we restrict our attention to the coevolution of populations belonging to two different species (prey and predator), so that, in general, the community is composed of $N_{1}$ prey populations and $N_{2}$ predator populations, with $N_{1}+N_{2}=N$. In particular, $n_{1}, \ldots, n_{N_{1}}$ and $x_{1} \leq \cdots \leq x_{N_{1}}$ are densities and traits of the $N_{1}$ prey populations, while $n_{N_{1}+1}, \ldots, n_{N_{1}+N_{2}}$ and $x_{N_{1}+1} \leq \cdots \leq x_{N_{1}+N_{2}}$ are densities and traits of the $N_{2}$ predator populations, respectively. Assume that initially $N_{1}=N_{2}=1$, so that $n_{1}$ and $x_{1}$ are prey density and trait while $n_{2}$ and $x_{2}$ are predator density and trait. At ecological timescale, the traits remain constant while the densities vary according to two ODEs of the form:

$$
\begin{aligned}
& \dot{n}_{1}=n_{1} F_{1}\left(n_{1}, n_{2}, x_{1}, x_{2}\right) \\
& \dot{n}_{2}=n_{2} F_{2}\left(n_{1}, n_{2}, x_{1}, x_{2}\right),
\end{aligned}
$$

where $F_{i}$ is the per capita growth rate of the $i$-th population. In the following, model (1), called resident model, is assumed to have one strictly positive and globally stable equilibrium $\bar{n}(x)=\left(\bar{n}_{1}(x), \bar{n}_{2}(x)\right)$ for each $x=\left(x_{1}, x_{2}\right)$ belonging to a set of the trait space called the evolution set of model (1).

At evolutionary timescale (slow dynamics), the traits vary according to two ODEs called evolutionary model of the form:

$$
\begin{aligned}
& \dot{x}_{1}=k_{1} G_{1}\left(x_{1}, x_{2}\right) \\
& \dot{x}_{2}=k_{2} G_{2}\left(x_{1}, x_{2}\right),
\end{aligned}
$$

where $k_{1}$ and $k_{2}$ are suitable constant parameters scaling the speed of evolution in the coevolving species determined by size and frequency of mutations. As mentioned above, population densities vary slowly with the traits, as model (1) is always at the equilibrium $\bar{n}(x)$ at evolutionary timescale.

The most transparent approach for deriving the evolutionary model (2) is the AD canonical equation [16, 4, 13]. It is based on the resident-mutant models, which describe the interactions among three populations, namely the two resident populations, and a mutant population with trait $x_{1}^{\prime}$ or $x_{2}^{\prime}$. When the mutation occurs in the prey, the prey population is split into two sub-populations (resident and mutant) with densities $n_{1}$ and $n_{1}^{\prime}$ and traits $x_{1}$ and $x_{1}^{\prime}$, so 
the model is:

$$
\begin{aligned}
& \dot{n}_{1}=n_{1} f_{1}\left(n_{1}, n_{2}, n_{1}^{\prime}, x_{1}, x_{2}, x_{1}^{\prime}\right) \\
& \dot{n}_{2}=n_{2} f_{2}\left(n_{1}, n_{2}, n_{1}^{\prime}, x_{1}, x_{2}, x_{1}^{\prime}\right) \\
& \dot{n}_{1}^{\prime}=n_{1}^{\prime} f_{1}^{\prime}\left(n_{1}, n_{2}, n_{1}^{\prime}, x_{1}, x_{2}, x_{1}^{\prime}\right),
\end{aligned}
$$

where functions $f_{1}, f_{1}^{\prime}$, and $f_{2}$ are consistently related to functions $F_{1}$ and $F_{2}$ (see [11, 13, chap. 3] for details). A similar three-dimensional model involving the mutant trait $x_{2}^{\prime}$, the density $n_{2}^{\prime}$, and a function $f_{2}^{\prime}$ describes the case in which the mutant is a predator. The values of $n_{1}^{\prime}$ and $n_{2}^{\prime}$ immediately after the mutation are very small because a mutant population is initially composed of one or a few individuals. In words, each mutation brings a new trait into the system, but competition between resident and mutant populations selects the trait that remains in the system in the long term. As long as mutants either disappear or substitute the corresponding residents, the evolutionary process is called mono-morphic — each species is present in a single morph - whereas di- and poly-morphic evolution phases follow after sequences of evolutionary branchings.

The mutation-competition process can be further specified by making suitable assumptions on the frequency and distribution of small mutations [16, 4, 13], and the conclusion is that the rate at which the trait $x_{i}$ varies at evolutionary timescale is given by the following ODE, called the AD canonical equation:

$$
\dot{x}_{i}=\left.k_{i} \bar{n}_{i}\left(x_{1}, x_{2}\right) \frac{\partial \lambda_{i}}{\partial x_{i}^{\prime}}\right|_{x_{i}^{\prime}=x_{i}},
$$

where $k_{i}$ is proportional to the frequency and size of mutations, $\bar{n}_{i}\left(x_{1}, x_{2}\right)$ is the equilibrium density of the resident model and $\lambda_{i}\left(x_{1}, x_{2}, x_{i}^{\prime}\right)=f_{i}^{\prime}\left(\bar{n}_{1}\left(x_{1}, x_{2}\right), \bar{n}_{2}\left(x_{1}, x_{2}\right), 0, x_{1}, x_{2}, x_{i}^{\prime}\right)$ is the so-called invasion fitness of the mutation (the initial exponential rate of increase of the mutant population). Equation (4), written for the prey and for the predator, gives the two ODEs that form the evolutionary model (2) with

$$
G_{i}\left(x_{1}, x_{2}\right)=\left.\bar{n}_{i}\left(x_{1}, x_{2}\right) \frac{\partial \lambda_{i}}{\partial x_{i}^{\prime}}\right|_{x_{i}^{\prime}=x_{i}} .
$$

Once the monomorphic dynamics has found a halt at a stable evolutionary equilibrium $\bar{x}=\left(\bar{x}_{1}, \bar{x}_{2}\right)$ of model (2), one can look at the second-order terms in the Taylor expansion of the mutant per capita growth rate to establish if the equilibrium is a branching point [27, 25,13 or not. More precisely, a stable equilibrium $\bar{x}$ of (2) is a branching point for the $i$-th population if

$$
B_{i}^{\prime}=\left.\frac{\partial^{2} \lambda_{i}}{\partial x_{i}^{\prime 2}}\right|_{\begin{array}{l}
x_{1}=\bar{x}_{1}, x_{2}=\bar{x}_{2}>0 \\
x_{i}^{\prime}=\bar{x}_{i}
\end{array}}>0
$$

and

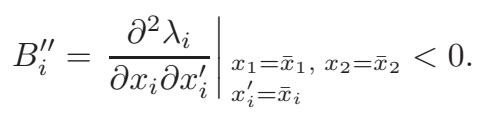

Under the latter condition, there exist a region in the plane $\left(x, x^{\prime}\right)$ (with the shape, locally to point $(\bar{x}, \bar{x})$, of a cone symmetrically opened with respect to the anti-diagonal, see [13, chap. 3]) for which small mutations in the $i$-th population invade and coexist, at a stable ecological equilibrium, with the former residents. The ecological equilibrium exists but is unstable if the condition is reversed; it does not exist if $B_{i}^{\prime \prime}=0$. 
Note that the computation of $B_{i}^{\prime \prime}$ requires the derivatives of the ecological equilibrium $\bar{n}(x)$, for which typically there is no analytical expression. These derivatives can however be computed by solving suitable systems of algebraic linear equations, as explained in Appendix.

Under condition (6), the two similar traits $x_{i}$ and $x_{i}^{\prime}$ further differentiate in accordance with the higher-dimensional canonical equation, where $x_{i}^{\prime}$ plays the role of a new resident trait. If conditions (6) and (7) are satisfied for both populations, i.e., for $i$ equal to 1 and 2 , each one will initially become dimorphic, but generically only one of the two nascent branchings survives. The reason is that the speed of divergence $\left|\dot{x}_{i}^{\prime}-\dot{x}_{i}\right|$ between the two branching morphs is given by $k_{i} \bar{n}_{i} B_{i}^{\prime}\left|x_{i}^{\prime}-x_{i}\right|+O\left(\left|x_{i}^{\prime}-x_{i}\right|^{2}\right)$ and (generically) differs among the two populations. Thus, as branching takes off (according to the four-dimensional canonical equation) in the population $i$ with largest exponential rate of divergence

$$
D_{i}^{\prime}=k_{i} \bar{n}_{i} B_{i}^{\prime},
$$

the evolution of $x_{i}$ and $x_{i}^{\prime}$ (generically) change the equilibrium trait $\bar{x}_{j}, j \neq i$, so that the pair $\left(x_{j}, x_{j}^{\prime}\right)$ falls outside the resident-mutant coexistence region that is present locally to point $\left(\bar{x}_{j}, \bar{x}_{j}\right)$ in the plane $\left(x_{j}, x_{j}^{\prime}\right)$ for the current values of $\left(x_{i}, x_{i}^{\prime}\right)$. Either the $x_{j}$ - or the $x_{j}^{\prime}$-population therefore goes extinct on the ecological timescale, so the population turns back monomorphic and branching does not develop (this phenomenon has been called "missed branching” in [37]).

In nongeneric cases, that is, for critical parameter combinations (e.g. on curves in parameter planes) or in models characterized by particular symmetries (see e.g. [49, 15, 20]), branching can develop simultaneously in more than one population. However, no symmetry is present in the specific coevolutioanry model we study in the next section, so that we restrain our attention to the branching scenarios that occur generically in parameter space.

Finally, if conditions (6) or (7) are not satisfied neither for $i=1$ nor $i=2$, no branching is possible and the equilibrium $\bar{x}$ is a terminal point (see [13, chap. 3]) of the evolutionary dynamics, among which the evolutionarily stable coalitions of game theory when $B_{i}^{\prime}$ is negative for all populations.

After a first branching has occurred, there are three resident populations, and one can repeat the analysis by considering the three corresponding resident-mutant models and by deriving from them the corresponding canonical equation. If the evolutionary trajectory originating at the branching point of the new canonical equation converges toward an equilibrium point, three different branchings are possible since there are three resident populations in the system. But the branching conditions are still based on the signs of $B_{i}^{\prime}$ and $B_{i}^{\prime \prime}$ (similarly specified as in (6) and (7)), where $i$ is the index spanning the resident populations $(i=1,2,3)$ and $\lambda_{i}\left(x_{1}, x_{2}, x_{3}, x_{i}^{\prime}\right)$ is the fitness of the mutants of the $i$-th population.

And the story continues like so through a sequence of successive evolutionary branchings (and possible evolutionary extinctions) until a terminal point is reached or the evolutionary trajectory tends toward a non-stationary (cyclic or chaotic) regime (called Red Queen behavior after [56], see also [45, 17, 44, 10, 14, 9]). In principle infinite branching sequences are possible, but have been shown to be structurally unstable, meaning that the region in parameter space associated to a possible sequence should shrink with the length of the sequence and vanish as the length diverges to infinity [29, 48].

A specific eco-evolutionary model. The ecological model we consider is the RosenzweigMacArthur prey-predator model [51]:

$$
\begin{aligned}
& \dot{n}_{1}=n_{1}\left(r-c n_{1}-\frac{a}{1+a h n_{1}} n_{2}\right) \\
& \dot{n}_{2}=n_{2}\left(e \frac{a n_{1}}{1+a h n_{1}}-d\right),
\end{aligned}
$$


where $r$ is prey per capita growth rate, $c$ is prey intraspecific competition, $a$ is predator attack rate, $h$ is predator handling time, namely the time needed by each predator to handle and digest one unit of prey, $e$ is predator efficiency, namely a conversion factor transforming each unit of predated biomass into predator newborns, and $d$ is predator death rate. The six positive parameters of the model $(r, c, a, h, e, d)$ could be reduced to three through rescaling. However, we do not follow this option because the biological interpretation of the dependence of the parameters on prey and predator traits would become less transparent.

In order to have a meaningful problem one must assume $e>d h$, because, otherwise, the predator population cannot grow even in the presence of an infinitely abundant prey population. For any meaningful parameter setting, model (9) has a global attractor in $\mathbb{R}_{+}^{2}$ [33], namely

(a) the trivial equilibrium $(r / c, 0)$ if $d / a(e-d h) \geq r / c$,

(b) a strictly positive equilibrium if $\frac{r a h-c}{2 a h c} \leq \frac{d}{a(e-d h)}<\frac{r}{c}$,

(c) a strictly positive limit cycle if $d / a(e-d h)<(r a h-c) /(2 a h c)$.

The transition from $(a)$ to $(b)$ is a transcritical bifurcation (which is generic in the class of positive systems of the form (9) [40]), while the transition from $(b)$ to $(c)$ is a supercritical Hopf bifurcation (see [40] for a proof). In the following, the functional dependencies of the parameters on the traits and the parameter values will be chosen to satisfy the condition for stationary coexistence (b). In particular we limit the value of the handling time $h$.

If we now imagine that a mutant population is also present, we can enlarge the resident model (9) by adding a third ODE and by modifying the equations of the resident populations in order to take the mutant population into account. Of course we also need to specify how the parameters depend upon the traits $x_{1}, x_{2}, x_{1}^{\prime}, x_{2}^{\prime}$. The number of possibilities is practically unlimited, because even for well identified prey and predator species there are many meaningful options. Thus, at this level of abstraction, it is reasonable to limit the number of parameters sensitive to the traits and avoid trait dependencies that could give rise to biologically unrealistic evolutionary dynamics, like the unlimited growth of a trait. Our choice has been to assume that the parameters $r, e$, and $d$ are independent on the traits, because this will allow us to compare our results with those obtained in [17] and [11]. Thus, in the case of a mutation in the prey population, the resident-mutant model is

$$
\begin{aligned}
\dot{n}_{1} & =n_{1}\left(r-c\left(x_{1}, x_{1}\right) n_{1}-c\left(x_{1}, x_{1}^{\prime}\right) n_{1}^{\prime}+\right. \\
& \left.-\frac{a\left(x_{1}, x_{2}\right)}{1+a\left(x_{1}, x_{2}\right) h\left(x_{1}, x_{2}\right) n_{1}+a\left(x_{1}^{\prime}, x_{2}\right) h\left(x_{1}^{\prime}, x_{2}\right) n_{1}^{\prime}} n_{2}\right) \\
\dot{n}_{2} & =n_{2}\left(e \frac{a\left(x_{1}, x_{2}\right) n_{1}+a\left(x_{1}^{\prime}, x_{2}\right) n_{1}^{\prime}}{1+a\left(x_{1}, x_{2}\right) h\left(x_{1}, x_{2}\right) n_{1}+a\left(x_{1}^{\prime}, x_{2}\right) h\left(x_{1}^{\prime}, x_{2}\right) n_{1}^{\prime}}-d\right) \\
\dot{n}_{1}^{\prime} & =n_{1}^{\prime}\left(r-c\left(x_{1}^{\prime}, x_{1}\right) n_{1}-c\left(x_{1}^{\prime}, x_{1}^{\prime}\right) n_{1}^{\prime}+\right. \\
& \left.-\frac{a\left(x_{1}^{\prime}, x_{2}\right)}{1+a\left(x_{1}, x_{2}\right) h\left(x_{1}, x_{2}\right) n_{1}+a\left(x_{1}^{\prime}, x_{2}\right) h\left(x_{1}^{\prime}, x_{2}\right) n_{1}^{\prime}} n_{2}\right) .
\end{aligned}
$$

If the mutation occurs in the predator population, the resident-mutant model involving the variables $n_{1}, n_{2}$, and $n_{2}^{\prime}$ and the traits $x_{1}, x_{2}$, and $x_{2}^{\prime}$ can be written in a similar way (see [11] for details).

We must now specify how the three remaining parameters, namely the prey intraspecific competition $c$, the predator attack rate $a$, and the predator handling time $h$, appearing in the resident-mutant models, depend on the resident and mutant traits. Due to our definition of 
the traits, which are scaled measures of the phenotypes, $c, a$, and $h$ are bounded functions. Unless otherwise stated, the parameters appearing in these functions are positive.

Prey intraspecific competition $c$ is given by

$$
c\left(x_{1}, x_{1}^{\prime}\right)=\frac{\gamma_{1}+\gamma_{2}\left(x_{1}-\gamma\right)^{2}}{1+\gamma_{0}\left(\gamma_{1}+\gamma_{2}\left(x_{1}-\gamma\right)^{2}\right)} \exp \left(-\left(\frac{x_{1}-x_{1}^{\prime}}{\gamma_{3}}\right)^{2}\right) .
$$

It is the product of two terms. The first defines the extra-mortality within groups of identical prey, that has a quadratic minimum at $x_{1}=\gamma$ and saturates at $1 / \gamma_{0}$ as $x_{1}$ diverges from $\gamma$ (the minimum competition and the local curvature are controlled by parameters $\gamma_{1}$ and $\gamma_{2}$, respectively). Parameter $\gamma$ (positive or negative) therefore describes a fixed characteristic of the environment, and is henceforth called optimum prey trait. The second term in (11) describes resident-mutant competition. Both resident and mutant prey suffer the highest (yet different) competition when they face identical individuals, as the exponential factor is maximum for $x_{1}=x_{1}^{\prime}$. The width $\gamma_{3}$ of this gaussian bell is inversely proportional to the sensitivity of competition to the resident-mutant phenotypic mismatch. High [low] sensitivity yields significant [mild] drops in competition as resident and mutant differentiate in phenotype. Intuitively, this suggests that prey branching might be favored by lowering $\gamma_{3}$, because the individuals of the less abundant prey population, whether resident or mutant, are more likely opposed to different rather than identical individuals, so they suffer a lower competition.

The predator attack rate $a$ is the bell-shaped function

$$
a\left(x_{1}-x_{2}\right)=\alpha_{0}+\alpha \exp \left(-\left|\frac{x_{1}-x_{2}}{\alpha_{1}}\right|^{2-\alpha_{2}}\right),
$$

where $\alpha_{1}$ and $\alpha_{2}$ are the width and the kurtosis of the function, respectively. In particular, if $0<\alpha_{2}<2$ the function is leptokurtic, if $\alpha_{2}<0$ the function is platykurtic, while $\alpha_{2}=0$ corresponds to the normokurtic function, i.e., a gaussian bell. Predator with pronounced $\alpha_{2}>0\left[\alpha_{2}<0\right]$ are called specialist [generalist] because they exploit a narrow [large] spectrum of prey. If prey and predator traits are tuned, that is, if $x_{1}=x_{2}$, the predator attack rate is maximum (and equal to $\alpha_{0}+\alpha$ ). When prey and predator traits are far from being tuned, the predator attack rate drops to $\alpha_{0}$. This supports the idea that in a system with one predator and two prey populations with diversified traits, the predator might be prone to branch into two different predator sub-populations with traits tuned with those of the two prey.

The predator handling time $h$ is the product of an increasing sigmoidal function of the prey trait $x_{1}$ and of a decreasing sigmoidal function of the predator trait $x_{2}$

$$
h\left(x_{1}, x_{2}\right)=\theta\left[1+\theta_{1}-\frac{2 \theta_{1}}{1+\exp \left(\theta_{3} x_{1}\right)}\right]\left[1+\theta_{2}-\frac{2 \theta_{2}}{1+\exp \left(-\theta_{4} x_{2}\right)}\right],
$$

where $\theta$ is the handling time corresponding to the tuned situation $x_{1}=x_{2}=0$.

The graphs of the functions $c\left(x_{1}, x_{1}^{\prime}\right), a\left(x_{1}-x_{2}\right)$, and $h\left(x_{1}, x_{2}\right)$ are shown in Fig. 1 for the parameter values indicated in the caption. Only the third of these functions, namely, the handling time $h$, coincides with those used in [11]. There are two reasons for this change. The first is that the functions proposed in this paper are biologically sound, while one of those used in [11], though fully appropriate for the purposes of that paper, was particularly extreme. In fact, in [11], the prey intraspecific competition corresponds to (11) for $\gamma_{3} \rightarrow \infty$, which means that the competition suffered by an individual only depends on its own trait and not on that of the opponent, a rather disputable biological assumption. The second reason is that the functions used in [11] produce very poor branching scenarios (at most one branching of the prey population), so that they were not suited for the purpose of this paper. 

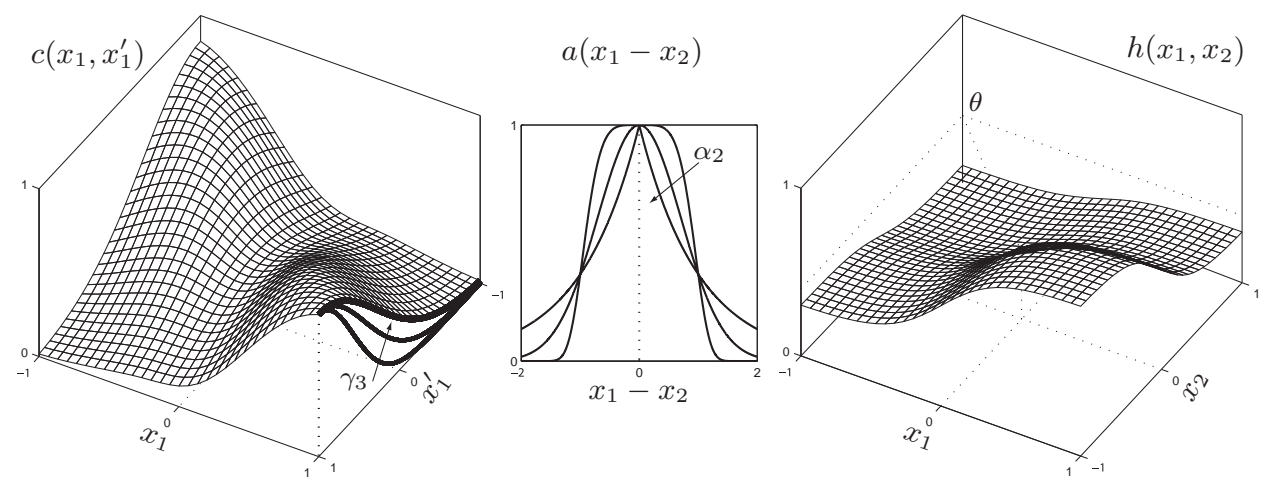

Fig. 1: Prey intraspecific competition $c$, predator attack rate $a$, and predator handling time $h$. The graphs correspond to the following parameter values: $\gamma=0, \gamma_{0}=1, \gamma_{1}=0.5$, $\gamma_{2}=5, \gamma_{3}=0.6,0.8,1, \alpha=1, \alpha_{0}=0, \alpha_{1}=1, \alpha_{2}=-3,0,1, \theta=0.4, \theta_{1}=\theta_{2}=0.5$, $\theta_{3}=\theta_{4}=5$.

Branching scenarios and branching sequences. So far, we have seen that the AD canonical equation (4) and the branching conditions (6) and (7) allow one to fully predict the evolution of biodiversity starting from a given ancestral community. Since the analysis presented in the next sections shows that evolutionary extinctions play no role in the coevolutionary model introduced in the previous section, we now focus our attention only on evolutionary branching.

Each branching scenario identifies a branching sequence, that is a sequence of symbols (1 and 2 in the case of two species), that specify in which species the branchings occur. It is important to notice that two different branching scenarios can be associated to the same branching sequence. For example, suppose that in a system initially composed of monomorphic prey and predator, a first branching occurs in the prey. If, after that, branching is again possible in both prey populations, we have two possible branching scenarios depending upon which one of the two prey does branch. However, the two branching scenarios identify the same branching sequence, namely the sequence $s=11$. In other words, branching sequences do not contain the full information on branching scenarios, but summarize the relevant information to study the evolution of biodiversity, namely the change in the number of coevolving prey and predator populations.

All branching sequences that can potentially occur in all eco-evolutionary models can be represented as paths from the root of the infinite binary tree $T$ shown in Fig. 2. The root node $(1,1)$ represents the ancestral community composed of two populations, one for each coevolving species, and the nodes of each layer $k=0,1,2, \ldots$ refer to communities with $k+2$ populations. Notice that finite sequences can be represented by the last node of the corresponding path in $T$, i.e., finite branching sequences and nodes of $T$ are interchangeable.

The aim of our analysis is to identify the branching sequences that develop from given ancestral conditions for different parameter values. Fixing the ancestral conditions means that we start from a given community (one prey, one predator in our case), characterized by given phenotypic traits $\left(x_{1}, x_{2}\right)$ and coexisting on a given ecological equilibrium $\bar{n}(x)$. Specifically, we analyze a compact domain $P$ of a parameter plane $\left(p_{1}, p_{2}\right)$.

For a transparent description of our approach we now give some definitions. Let $s$ be a finite branching sequence, which could be the first part of a longer sequence (called $s$ - 


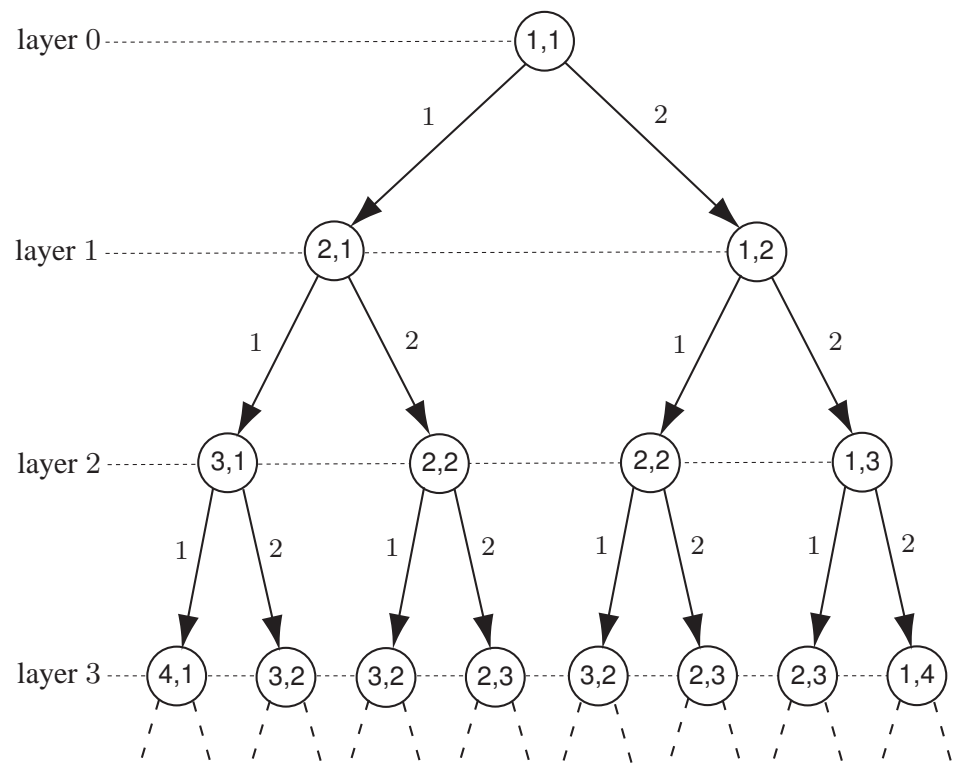

Fig. 2: The tree $T$ representing all potential branching sequences of a two-species community. Each node $\left(N_{1}, N_{2}\right)$ represents a community composed of $N_{1}$ prey populations and $N_{2}$ predator populations and belongs to the layer $k=N_{1}+N_{2}-2, k=0,1,2, \ldots$. Edges 1 and 2 represent prey and predator branching, respectively.

extension). A branching sequence $s$ is called observable iff there exists $p=\left(p_{1}, p_{2}\right) \in P$ that produces a branching scenario associated to $s$ or to an $s$-extension. The subset of $P$ giving rise to an observable branching sequence $s$ is indicated by $O(s)$.

An observable sequence $s$ is called complete iff there exists $p \in O(s)$ that does not produce an $s$-extension; incomplete otherwise. The subset of $O(s)$ giving rise to a complete branching sequence $s$ is indicated with $C(s)$.

By definition, given a branching sequence $s$, we have

$$
\varnothing \subseteq C(s) \subseteq O(s) \subseteq P .
$$

If $O(s)=\varnothing$ the branching sequence $s$ cannot occur and the corresponding node in $T$ can be eliminated together with all its successors.

If $O(s) \neq \varnothing$ and $C(s)=\varnothing$ the branching sequence $s$ is incomplete and the corresponding node in the tree can be identified with a particular color (grey). In this case, $s$-extensions can be of any type because through extension one can obtain completeness or not, or even lose observability.

If $C(s) \neq \varnothing$ the branching sequence $s$ is complete and the corresponding node in the tree can be identified with a second color (white). Again, the successors of a white node in the tree can be of any type.

When all nodes of $T$ are eliminated or colored in the way just described, a new tree $B T$, called branching tree, is obtained. Notice that the tree $B T$ can be infinite (but recall that infinite sequences require some criticality in the choice of the model functions and parameters $[29,48])$. Moreover, a non empty subset of $P$ can be associated to each node of the branching tree: the set $O(s)$ to each gray node identifying the incomplete sequence $s$; the set $C(s)$ to 

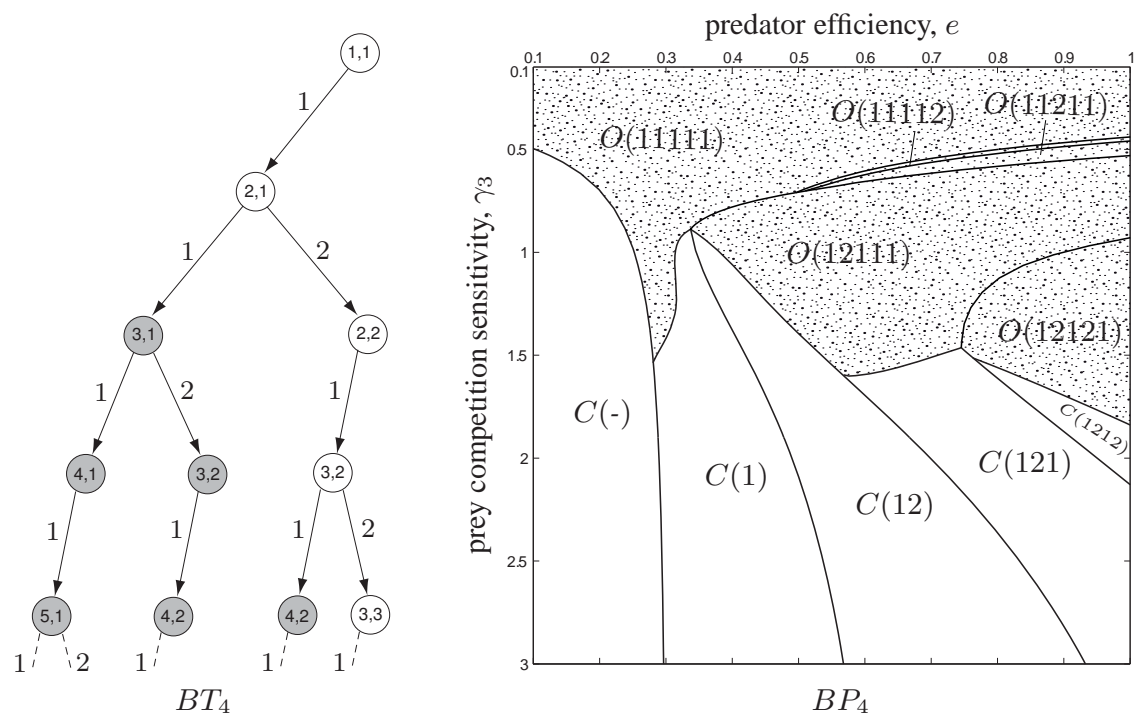

Fig. 3: The tree $B T_{4}$, where white and grey nodes represent complete and incomplete branching sequences. The approximation $\mathrm{BP}_{4}$ of the branching portrait, where a complete branching sequence of length smaller or equal to 4 is associated to each white region, whereas a sequence of length equal to 5 is associated to each dotted region. All parameters, except $e$ and $\gamma_{3}$, are at their reference values: $r=0.5, d=0.05, \gamma=0, \gamma_{0}=0.01, \gamma_{1}=0.5$, $\gamma_{2}=2.3, \alpha=1, \alpha_{0}=0.01, \alpha_{1}=1, \alpha_{2}=0, \theta=\theta_{1}=\theta_{2}=0.5, \theta_{3}=\theta_{4}=1$, $k_{1}=k_{2}=1$. The considered ancestral condition is $x_{1}(0)=0, x_{2}(0)=0$.

each white node identifying the complete sequence $s$. The sets $C(s)$ are all disjoint, whereas given two sequences $s^{\prime}$ and $s^{\prime \prime}, O\left(s^{\prime \prime}\right) \subseteq O\left(s^{\prime}\right)$ iff $s^{\prime \prime}$ extends $s^{\prime}$. The collection $C$ of all sets $C(s)$ obviously defines a partition of the parametric domain $P$, though some of the sets $C(s)$ may have zero measure in $P$ (those corresponding to non-generic sequences, e.g. sequences where several branching occur simultaneously).

Finally, we call branching portrait $(B P)$ the diagram of the collection $C$. This parametric portrait graphically summarizes the analysis of branching scenarios and can be used as a control chart in deriving managerial policies. It can be composed of infinite regions, though shrinking with the length of the sequence, so that an iterative procedure for its construction, that considers sequences with increasing length, is proposed in the next section.

Iterative procedure. In this section we show how the branching portrait $B P$ can be progressively approximated by applying an iterative procedure involving simulations and continuations. The procedure is illustrated by applying it to the prey-predator eco-evolutionary model described above. The two parameters $p_{1}$ and $p_{2}$, belonging to two given intervals, are $e$ and $\gamma_{3}$, i.e., predator efficiency (see (9)) and the parameter specifying the sensitivity of prey intraspecific competition to resident-mutant phenotypic mismatch (see (11)).

The procedures generates at iteration $k$ the tree $B T_{k}$, composed of layers $0, \ldots, k$ of the branching tree $B T$, and an approximation $B P_{k}$ of the branching portrait $B P$. Specifically, $B P_{k}$ includes the sets $C(s)$ associated to branching sequences $s$ of length smaller or equal to $k$, while the remaining area of the parameter domain $P$ is divided into the sets $O(s)$ associated to sequences of length equal to $k+1$. All this information can be obtained by analyzing the 
canonical equation describing the communities corresponding to the nodes at layer $k$ of $B T_{k}$. In particular, the branching points in these communities identify the observable sequences of length $k+1$ and define the nodes at layer $k+1$ of $B T_{k+1}$, their color to be determined at the next iteration.

For example, in the case of our prey-predator model, for $k=4$, the tree $B T_{4}$ and the portrait $\mathrm{BP}_{4}$ are as in Fig. 3. The white region $C_{4}=C(-) \cup C(1) \cup C(12) \cup C(121) \cup C(1212)$ in $\mathrm{BP}_{4}$ is the collection of the sets $C(s)$ of the white nodes of $B T_{4}$, i.e., the sets $C(s)$ of all complete branching sequences of length smaller or equal to $k=4$. Hence, $C_{4} \subseteq C$. The dotted region is the union of the sets $O(s)$ of sequences of length $k+1=5$, so five branchings are observable in this region. Obviously, the dotted region shrinks and eventually disappears as $k$ is increased. In conclusion, smaller dotted regions in $B P_{k}$ correspond to better approximations of $B P$.

Iteration 0. We start with $k=0$, i.e., with a degenerate tree composed of the root node $(1,1)$ corresponding to the empty sequence $s=-$, and the target is to determine $B T_{0}$ and $B P_{0}$. As for $B T_{0}$, we must establish the nature (complete or not) of the root node, whereas for $B P_{0}$, we must determine the set $C_{0}=C(-)$, i.e., the parameter values for which no branching is possible, and the sets $O(1)$ and $O(2)$ where sequences of length $k+1=1$ are observable.

For this, we start our analysis from a given point in the parameter domain $P$ (we start from $\left.p=\left(e, \gamma_{3}\right)=(0.1,2)\right)$ and determine the stable equilibrium $\left(\bar{x}_{1}, \bar{x}_{2}\right)$ of the corresponding $\mathrm{AD}$ canonical equation (4) reached from the considered ancestral condition. This can be easily accomplished in a standard way, because the non-trivial equilibrium $\left(\bar{n}_{1}\left(x_{1}, x_{2}\right), \bar{n}_{2}\left(x_{1}, x_{2}\right)\right)$ of the ecological model (9) is known in closed form (see [11]). However, this is not true for the nodes of all larger trees, because the equilibrium $\bar{n}(x)$ of the resident model cannot be derived analytically when the populations are three or more. Thus, we systematically determine the stable equilibrium of any $\mathrm{AD}$ canonical equation through the simulation of an eco-evolutionary model characterized by two different time scales. In the case $k=0$ the slow-fast ODE system is

$$
\begin{aligned}
& \dot{n}_{1}=n_{1} F_{1}\left(n_{1}, n_{2}, x_{1}, x_{2}, e, \gamma_{3}\right) \\
& \dot{n}_{2}=n_{2} F_{2}\left(n_{1}, n_{2}, x_{1}, x_{2}, e, \gamma_{3}\right) \\
& \dot{x}_{1}=\epsilon k_{1} G_{1}\left(x_{1}, x_{2}, e, \gamma_{3}\right) \\
& \dot{x}_{2}=\epsilon k_{2} G_{2}\left(x_{1}, x_{2}, e, \gamma_{3}\right),
\end{aligned}
$$

where $\epsilon$ is a small positive parameter used to tune the slow evolutionary dynamics with respect to the fast ecological dynamics (in our application the value $\epsilon=10^{-3}$ has given satisfactory results).

For $p=\left(e, \gamma_{3}\right)=(0.1,2)$ and the ancestral condition we consider $\left(x_{1}(0)=0, x_{2}(0)=\right.$ 0 ), system (15) tends toward the stable equilibrium $\left(\bar{n}_{1}, \bar{n}_{2}, \bar{x}_{1}, \bar{x}_{2}\right)=(0.6589,0.2269,-0.0048,0.0371)$, thus characterized by

$$
F_{1}=F_{2}=G_{1}=G_{2}=0 .
$$

Once this equilibrium has been found, it is possible to continue it by varying $e\left[\gamma_{3}\right]$. The aim is to continue it until a point where $B_{1}^{\prime}=0$ is obtained, that is, until a solution of the system with five equations

$$
F_{1}=F_{2}=G_{1}=G_{2}=B_{1}^{\prime}=0
$$

is found. Since the unknowns in this system are six $\left(n_{1}, n_{2}, x_{1}, x_{2}, e, \gamma_{3}\right)$, the solution of (17) can in turn be continued by varying both $e$ and $\gamma_{3}$, thus finding a curve $e\left(\gamma_{3}\right)\left[\gamma_{3}(e)\right]$ in 
parameter space. On this curve, shown in $B P_{0}$ in Fig. 4, the first branching function of the prey $B_{1}^{\prime}$ annihilates. Therefore, at one side of the curve the first branching condition for the prey ((6) with $i=1)$ is satisfied.

In principle, analogous operations should be done for the other three branching functions $B_{1}^{\prime \prime}, B_{2}^{\prime}$ and $B_{2}^{\prime \prime}$ to possibly obtain other three curves in parameter space. The region in which population $i$ does branch would then be the one in which $B_{i}^{\prime}>0$ and $B_{i}^{\prime \prime}<0$, provided population $i$ has the largest resident-mutant trait divergence (see (8)) where both prey and predator satisfy the branching conditions (6) and (7). However, it is possible to numerically verify that $B_{1}^{\prime \prime}$ is always negative (see Appendix). This property is valid in general, that is, also in systems with many populations of prey and predator, and is due to the exponential term in the competition function (11) which favors resident-mutant coexistence. Hence, the branching condition of the $i$-th prey population is $B_{i}^{\prime}>0$.

Instead, for the predator, the function $B_{2}^{\prime \prime}$ is identically null, so that the predator branching is not possible. Also this property is valid in general, when the number of predator populations $\left(N_{2}\right)$ is equal to the number of prey populations $\left(N_{1}\right)$. As already noticed in [11], this is a direct consequence of the well known competitive exclusion principle [31, 42, 43, 41] and its more recent generalizations [18, 19, 29, 48]. If, otherwise, $N_{1}>N_{2}$, then $B_{i}^{\prime \prime}$ is negative for all predator populations (numerically checked), so that, the branching condition for the $i$-th predator population reduces to $B_{i}^{\prime}>0$. Using the terminology introduced in [18], the number of stationary coexisting morphs of a species is limited by the number of biotic environmental factors affecting the ecological dynamics of the species. For the predator, these factors are simply the prey densities, i.e., $N_{1}$ factors.

While monitoring functions $B_{1}^{\prime}$ and $B_{2}^{\prime}$ during continuation, we must also check whether the equilibrium of system (15) undergoes a bifurcation [40, 47], e.g. it might disappear through a fold bifurcation or lose stability through a Hopf bifurcation. This is easy and automatically done by standard continuation softwares. In the first case, the evolutionary trajectory originated from the considered ancestral condition will converge to another evolutionary attractor, possibly an evolutionary equilibrium that might generate a different branching sequence, whereas evolutionary cycling prevents further branching in the second case. Recall that our choice of the model parameters guarantees the stationary coexistence of the demographic model (1), so that bifurcations can only involve the slow dynamics of system (15). Moreover, while moving parameters in the presence of multiple evolutionary attractors, different branching sequences might also arise without bifurcations, simply because the considered ancestral condition switches from the basin of attraction of one attractor to that of another. Multi-stability is related to the presence of saddle equilibria, whose stable manifolds are the boundaries of the different basins of attraction. Since attractors themselves, saddles, and their manifolds move in the state space when changing parameters, it could happen that the considered ancestral condition (which is fixed and does not change with parameters) passes, at a critical parameter value, from the basin of attraction of one attractor to that of another. Technically, this is not a bifurcation, but implies a qualitatively different evolutionary dynamics.

None of the above possibilities occurred in system (15), whereas fold bifurcations will be found in the further iterations of the procedure, though with no effect on the branching portraits. For this reason, they will no more be discussed in the next iterations. Otherwise, Hopf bifurcations will be found and will affect the branching portraits with respect to other parameter pairs that will be presented in the Discussion.

In conclusion, the iteration $k=0$ of the procedure shows that the empty branching sequence $s=-$ is complete, because region $C(-)$ (where no branching is possible) is not empty, so that the color of the root of the tree $B T_{0}$ (the first node of $B T_{4}$ in Fig. 3) is white. 

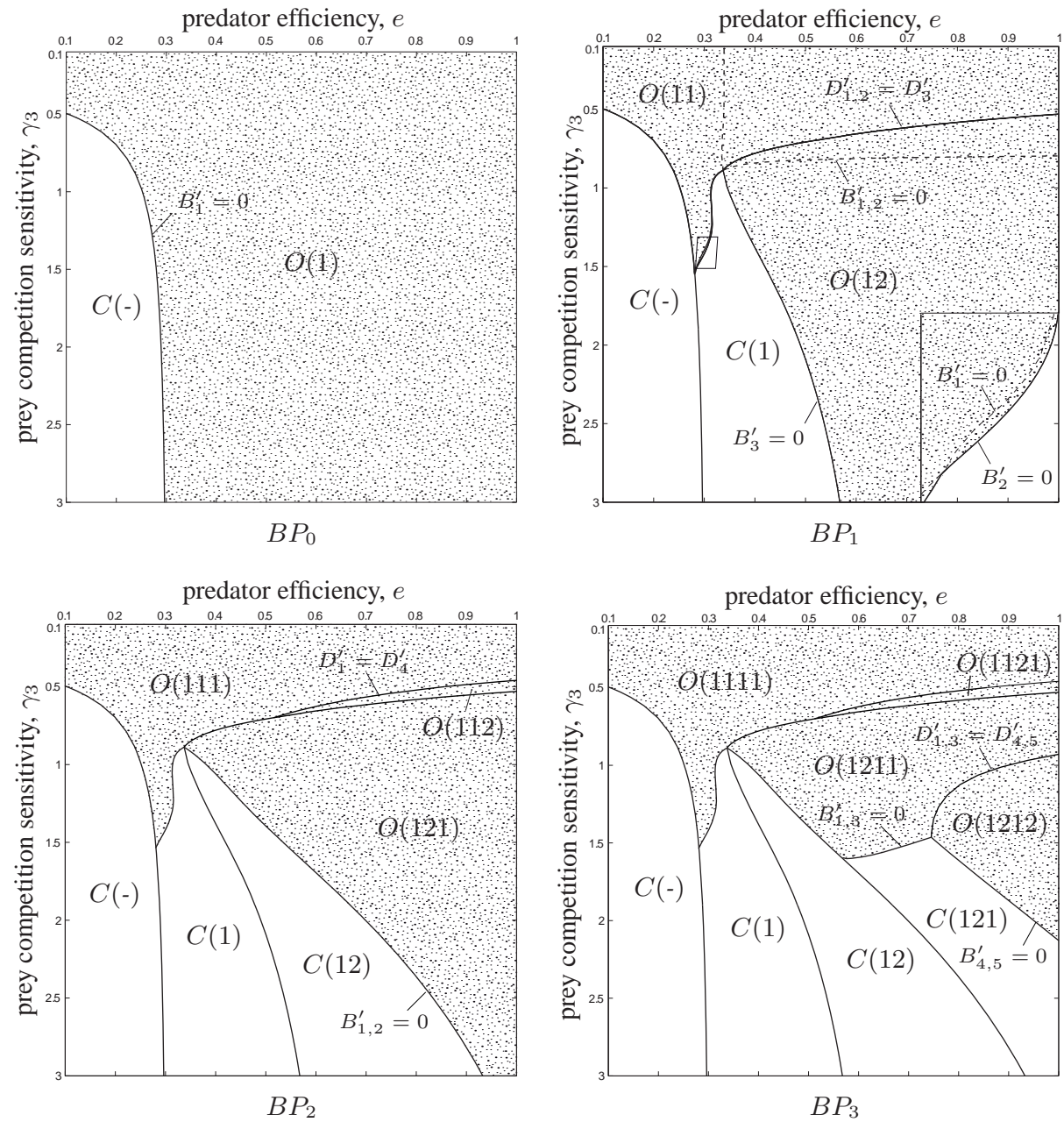

Fig. 4: The approximated branching portraits $B P_{0}-B P_{3}$ produced at the iterations $k=$ $0,1,2,3$ of our procedure. At each iteration the boundaries added to the diagram are curves of the type $B_{i}^{\prime}=0$ or $D_{i}^{\prime}=D_{j}^{\prime}$ and are accordingly labeled (recall that at iteration $k$ we analyze communities with $N_{1}+N_{2}=k+2$ populations and that $i=1, \ldots, N_{1}$ and $i=N_{1}+1, \ldots, N_{1}+N_{2}$ are prey and predator indexes, respectively). As shown in $B P_{1}$ (see also enlarged box), the region boundaries (solid lines) might concatenate segments of different curves. Parameter values as in Fig. 3.

The first approximation $B P_{0}$ of the branching portrait $B P$ is shown in Fig. 4, where the dotted region is nothing but $O(1)$, since $O(2)$ is empty because the predator cannot branch (so that $O(1)=P-C(-)$ ).

Iteration 1. At the iteration $k=1$ the community has two prey populations $\left(N_{1}=2\right)$ and one predator population $\left(N_{2}=1\right)$ and the target is to determine $B T_{1}$ and $B P_{1} . B T_{1}$ has only node $(2,1)$ at level 1 (because predator branching is not possible at the root node), so we must establish its nature. As for $B P_{1}$, we must determine the set $C(1)$, i.e., the parameter 
values for which no branching is possible at node $(2,1)$, and the sets $O(11)$ and $O(12)$ where sequences of length $k+1=2$ are observable. Of course, such sets are contained in $O(1)$, i.e., in the dotted region of $B P_{0}$ (see Fig. 4).

For this, first we write the AD canonical equation (composed of three ODEs, one for each population), and then determine its stable equilibrium $\left(\bar{x}_{1}, \bar{x}_{2}, \bar{x}_{3}\right)$. Similarly to what was done in the previous subsection, we simulate a slow-fast eco-evolutionary system with six variables: $n_{1}, n_{2}, n_{3}$ and $x_{1}, x_{2}, x_{3}$, where $n_{3}$ and $x_{3}$ are predator density and trait. This system is analogous to (15) and must be simulated starting from initial conditions that represent the state of the system just after a prey branching has occurred in the system with $N_{1}=N_{2}=1$. This links this iteration with the previous one. For producing the six required initial conditions we select any point on the curve $B_{1}^{\prime}=0$ in $B P_{0}$, which corresponds to a stable equilibrium of system (15) and we continue it by increasing $e$ in order to enter into the dotted region $O(1)$ where prey branching occurs. Since close to the branching point the two similar traits coexist and evolve in opposite directions, we define the initial condition as follows: $n_{1}(0)=n_{2}(0)=\bar{n}_{1} / 2, n_{3}(0)=\bar{n}_{2}, x_{1}(0)=\bar{x}_{1}-\delta, x_{2}(0)=\bar{x}_{1}+\delta, x_{3}(0)=\bar{x}_{2}$, where $\left(\bar{n}_{1}, \bar{n}_{2}, \bar{x}_{1}, \bar{x}_{2}\right)$ is the obtained equilibrium of system (15) (we used $\delta=10^{-3}$ ). Once a stable equilibrium $\left(\bar{n}_{1}, \bar{n}_{2}, \bar{n}_{3}, \bar{x}_{1}, \bar{x}_{2}, \bar{x}_{3}\right)$ has been obtained by simulating the six-dimensional eco-evolutionary system, the parameter $e$ and/or $\gamma_{3}$ are varied until three points in parameter space are obtained where $B_{1}^{\prime}=0, B_{2}^{\prime}=0, B_{3}^{\prime}=0$, respectively. These three points belong to the boundaries of the regions where populations $1,2,3$ can branch, so that the three boundaries can be produced through continuation of the solution of the algebraic systems

$$
F_{1}=F_{2}=F_{3}=G_{1}=G_{2}=G_{3}=B_{i}^{\prime}=0
$$

with $i=1,2,3$.

The result allows one to determine, by simply looking at the signs of all $B_{i}^{\prime}$ in the vicinity of the curves, the tree $B T_{1}$ (the first two layers in $B T_{4}$ in Fig. 3) and the approximation $B P_{1}$ of the branching portrait (see Fig. 4). Node $(2,1)$ is white because region $C(1)$ (where one and only one prey branching occurs) is not empty. The dotted region of $B P_{1}$, to be further investigated, is the union of the sets $O(11)$ and $O(12)$, where prey or predator branching occurs at node $(2,1)$. Note that the curves $B_{1}^{\prime}=0$ and $B_{2}^{\prime}=0$ are very close one to the other and not distinguishable at the scale of the figure. The enlargement in $B P_{1}$ shows that $O(11)$ is the region where at least one of the two prey can branch. Where both prey can branch, it does not matter which one does it — the one with faster resident-mutant divergence (see (8)) — since further branchings produce the same sequences. Where also the predator acquires a positive $B_{3}^{\prime}$, the population that branches is again the one with faster resident-mutant divergence, and in this case the lines along which $D_{1}^{\prime}=D_{3}^{\prime}$ and $D_{2}^{\prime}=D_{3}^{\prime}$ (again not distinguishable at the scale of the figure) matter in the branching portrait.

Successive iterations. The successive iterations proceed along the same lines we have described in detail in the two previous subsections. We now briefly summarize seven sequential steps in which each iteration can be subdivided. We recall that the target of iteration $k$ is the determination of the tree $B T_{k}$ and of the approximation $B P_{k}$ of the branching portrait $B P$.

1. Write the $\mathrm{AD}$ canonical equation associated to each of the sets $O(s)$, with $s$ of length $k$, composing the dotted region of the approximation $B P_{k-1}$ of the branching portrait determined at the previous iteration. In each of these regions, after the first $k$ branchings, the community is composed of $N_{1}$ prey and $N_{2}$ predator (with $N_{1}+$ $N_{2}=k+2$ ). For example, at the iteration 2, we consider the sets $O(11)$ with $N_{1}=3$ and $N_{2}=1$ and $O(12)$ with $N_{1}=2$ and $N_{2}=2$ (see $B P_{1}$ in Fig. 4). 


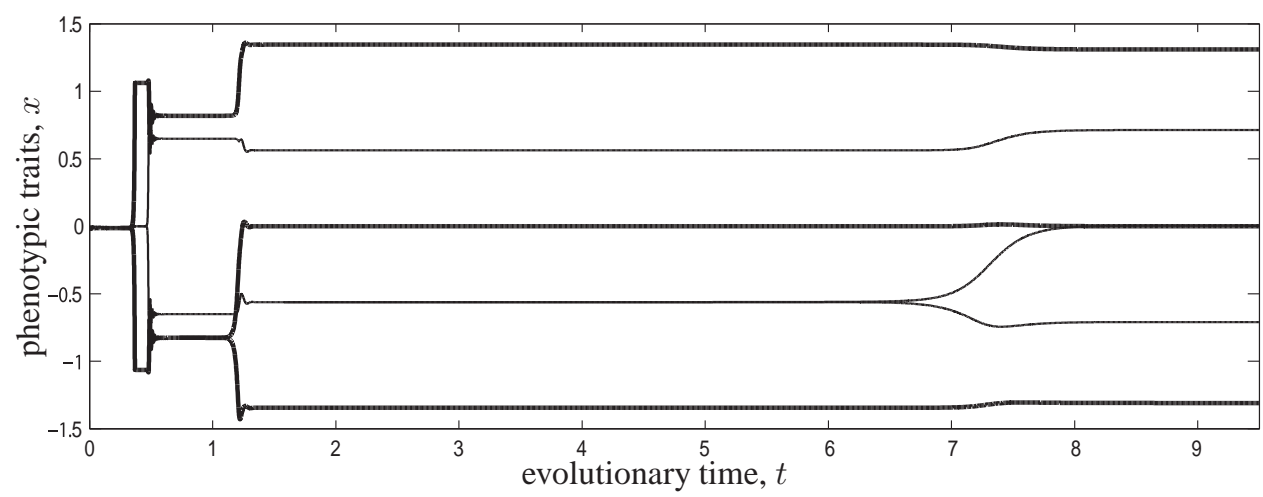

Fig. 5: Simulation of successive AD canonical equations for the alternate branching sequence $s=1212$. Thick (resp. normal) line: prey (resp. predator) traits. Parameter values are as in Fig. 3, $e=0.98$ and $\gamma_{3}=2$. Branching instants are chosen when $\dot{x}_{i}<10^{-9}$ for each $i$.

2. For each considered canonical equation, write the corresponding $2(k+2)$-dimensional slow-fast eco-evolutionary system.

3. Determine the stable equilibrium of the eco-evolutionary system through simulation. The initial conditions of the simulation must represent the density and the trait of the populations just after the $k$-th branching. Note that if the boundary of the set $O(s)$ is composed of several segments corresponding to branching of different populations, an initial condition for each branching must be considered.

4. Continue the stable equilibria of the eco-evolutionary systems varying $e$ or $\gamma_{3}$ until a point on each possible curve where $B_{i}^{\prime}=0$ is found. If no such point is found, $B_{i}^{\prime}$ never changes sign. If $B_{i}^{\prime}$ is negative, no branching is possible for population $i$, whereas if it is positive branching is possible in the whole region.

5. Produce the curves on which $B_{i}^{\prime}=0$ through continuation of the points determined at step 4.

6. In the regions where $B_{i}^{\prime}$ and $B_{j}^{\prime}$ are positive for $i \neq j$, produce through continuation the curves on which $D_{i}^{\prime}=D_{j}^{\prime}$.

7. Determine (through a simple inspection of the signs of $B_{i}^{\prime}$ near the curves) the tree $B T_{k}$ and the approximation $B P_{k}$ of the branching portrait $B P$. In particular, $C_{k}$ is obtained by adding to $C_{k-1}$ the parameter combinations for which no further branching has been detected, whereas the new dotted region is the union of the identified sets $O(s)$, with $s$ of length $k+1$. The boundary of such sets are obtained by suitable concatenating segments of the obtained curves $B_{i}^{\prime}=0$ and $D_{i}^{\prime}=D_{j}^{\prime}$.

Fig. 4 shows the approximations $B P_{k}$ of the branching portrait obtained for $k=0,1,2,3$ while $\mathrm{BP}_{4}$ was already reported in Fig. 3. The comparison of these approximations clearly points out that our procedure is rather efficient since the dotted region (that must be further analyzed at iteration $k+1$ ) shrinks significantly at each iteration.

Fig. 5 shows an example of simulation of successive canonical equations corresponding to a complete sequence with four alternate branchings.

Discussion. In the first part of this section we discuss the main biological consequences of the analysis performed so far, while in the second part we show how, with a marginal extra computational effort, the scope of the analysis can be substantially enlarged. Finally, we describe the steps that are required to abstract from our analysis and propose a general 
method for investigating branching scenarios in coevolving species.

Before discussing the approximation of the branching portrait in Fig. 3, let us notice that the parameter $\gamma_{3}$ on the vertical axis of that figure is increasing from top to bottom. Since decreasing values of $\gamma_{3}$ correspond (see (11)) to increasing sensitivities of prey intraspecific competition to the resident-mutant phenotypic mismatch, in interpreting Fig. 3 we must take into account that its left lower corner represents prey with low sensitivity of competition and predator with low efficiency, whereas highly sensitive competing prey and very efficient predator are located at the right upper corner.

The first property emerging from Fig. 3 is that the region $C(-)$ where branching is not possible is in the left lower corner. This is quite intuitive from a biological point of view, because prey with low sensitivity of competition has no relevant advantages in splitting when the predation pressure is limited by a low predator efficiency. For the same reason, at the right upper corner, long branching sequences are possible.

A second interesting property pointed out by Fig. 3 is that both alternate (prey and predator) and unilateral (only prey) branching sequences are possible. More precisely, long alternate branching sequences are favored by high predator efficiency, while long unilateral prey branching sequences (see for example the set $O(11111)$ ) occur for low predator efficiency and high prey sensitivity of competition. Also this property can be intuitively understood because when prey competition is highly sensitive prey have an advantage in splitting in order to reduce the negative consequences of being too similar.

Notice that also long branching sequences composed of a first phase of alternate branching concatenated with a long sequence of prey branching are possible (see e.g. region $O(12111)$ in Fig. 3), but require a few more iterations of our procedure in order to be clearly pointed out in better approximations of the branching portrait (e.g. observable prey branchings are added to $O(12121)$ in $B P_{k}$ for $k>4$ ).

Finally, a third property that is worth mentioning is that branching scenarios can be highly sensitive to parameter perturbations. This is clearly visible close to the points in parameter space where two or more region boundaries merge. Also the boundary separating set $C(-)$ from $O(11111)$ in $B P_{4}$ shows that as soon as prey branching becomes possible at node $(1,1)$, branching is possible also at nodes $\left(N_{1}, 1\right)$, with $N_{1} \geq 2$, so that a small parameter perturbation can discriminate between very poor and extremely rich prey biodiversity. This latter property has also been observed in the Lotka-Volterra competition model [37, 7].

In conclusion, in coevolving prey-predator systems not only the number of prey populations (i.e., biodiversity) is higher than the number of predator populations (as implied by the principle of competitive exclusion) but often this difference is remarkable, in particular when prey intraspecific competition is highly sensitive to the resident-mutant phenotypic mismatch. This conclusion is in good agreement with many studies based on field observations of aquatic and terrestrial food chains like phytoplankton-zooplankton in shallow lakes [53], rodents and their predators in boreal and arctic regions [55, 36], and many others [3].

We now show how the analysis described so far, concerning the influence of two parameters $p_{1}$ and $p_{2}$ on branching scenarios, can be extended to study the influence of any other parameter $p_{3}$. The idea, suggested by the power and flexibility of continuation methods, is very simple. Suppose an approximation of the branching portrait $B P$, like that shown in Fig. 3, has already been produced. Except for $p_{1}$ and $p_{2}$, this approximated portrait has been computed for fixed reference values

$$
p_{i}=p_{i}^{*} \quad i=3,4,5, \ldots
$$

of all other parameters (see the caption of Fig. 3). We can therefore fix $p_{2}$ at a particular reference value $p_{2}^{*}$ and read from $B P_{4}$ in Fig. 3 the $p_{1}$-coordinates of all points of the boundaries 
of the various regions with $p_{2}=p_{2}^{*}$. Obviously, these $p_{1}$-coordinates allow one to determine in the space $\left(p_{1}, p_{3}\right)$ a series of points with $p_{3}=p_{3}^{*}$ belonging to the boundaries of the various regions of the approximated branching portrait obtainable for

$$
p_{i}=p_{i}^{*} \quad i=2,4,5, \ldots
$$

Then, with a limited computational effort, we can produce through continuation of this series of points a new approximated branching portrait in the space $\left(p_{1}, p_{3}\right)$. This means that, with almost the same computational burden necessary for discussing the influence of a pair of parameters, we can, in reality, discuss the influence of any other parameter pair.

Six examples of new branching portraits obtained from Fig. 3 by fixing $\gamma_{3}=2$ are shown in Fig. 6. Since the branching sequences obtained for $\gamma_{3}=2$ are all complete in $\mathrm{BP}_{4}$, it was reasonable to expect exact branching portraits in the space $\left(p_{1}, p_{3}\right)$ (no dotted regions) characterized by complete sequences, at least for small deviations of $p_{3}$ from $p_{3}^{*}$. All branching portraits have predator efficiency on their horizontal axis (as $\mathrm{BP}_{4}$ in Fig. 3) while the new parameter on the vertical axis is

(a) the ratio $k_{1} / k_{2}$ of the evolution speed of prey and predator (see (2)),

(b) the predator handling time $\theta$ (see (13)),

(c) the optimum prey trait $\gamma$ (see (11)),

(d) the curvature of prey competition $\gamma_{2}$ around the optimum prey trait (see (11)),

(e) the predator maximum attack rate $\alpha$ (see (12)),

(f) the kurtosis of predator attack rate $\alpha_{2}$ (see (12)).

The first five new parameters have been selected in order to obtain results comparable with those reported in [11], while the sixth choice has been suggested by the interest in discussing the role played by generalist vs. specialist predator on evolution (see [52, 32, 50]).

Fig. 6(a) shows that the ratio of evolution speed has no influence on branching scenarios. This might seem obvious a priori, since $k_{1}$ and $k_{2}$ do not modify the evolutionary equilibria and the branching conditions (6) and (7). However, the ratio $k_{1} / k_{2}$ affects the stability of the evolutionary equilibria (recall that branching points have been defined as stable equilibria of the canonical equation satisfying conditions (6) and (7)), though no change in stability occurs in Fig. 6(a).

Fig. 6(b) shows that the predator handling time $\theta$ has a detectable impact on branching scenarios. In particular, for fixed predator efficiency, the number of alternate branchings decreases if $\theta$ is increased. As far as we know, this property has never been discussed in the literature, though it is biologically sensible: predators with longer handling times exert a limited predation pressure and therefore do not turn selection disruptive on prey with relatively low sensitivity of competition.

Fig. 6(c) shows that the optimum prey trait $\gamma$ has almost no influence on branching scenarios. This is perhaps intuitive because a variation of $\gamma$ essentially introduces a shift in the body size of the prey (and hence of the predator). In our model, however, the handling time is sensitive to such a shift, so that there is an influence (branching being slightly favored by nonzero values of $\gamma$ ), though not clearly visible at the scale of the figure.

In contrast, the influence of the prey competition curvature $\gamma_{2}$ (Fig. 6(d)) is significant. This property seems also to have gone unnoticed in the literature and is less intuitive. A smaller curvature implies a larger valley of the competition function around the optimum prey trait $\gamma$, i.e. a larger trait interval that prey can exploit through branching for escaping the predation pressure; and predator branching is favored after each prey diversification. This mechanism however does not work if competition is too mild, as the evolutionary dynamics in the system with three prey and two predator populations get destabilized. The five coevolving traits start to oscillate on a Red Queen evolutionary cycle, along which the two predators 


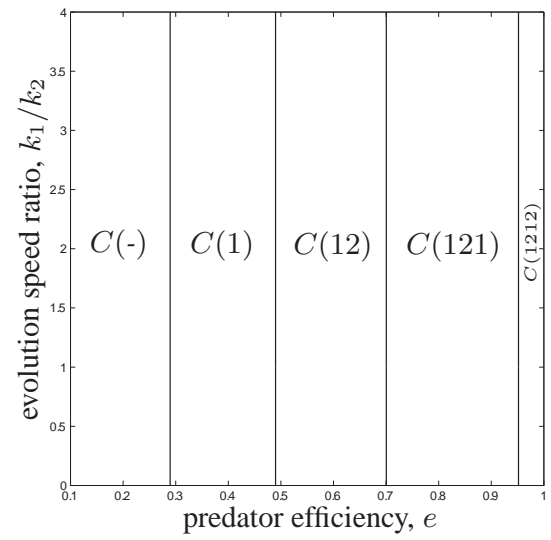

(a)

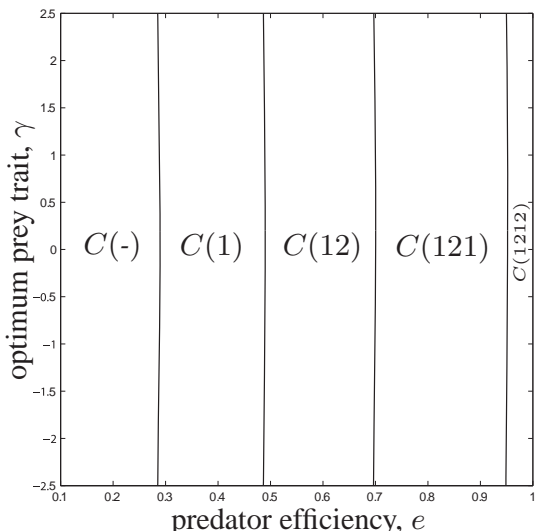

(c)

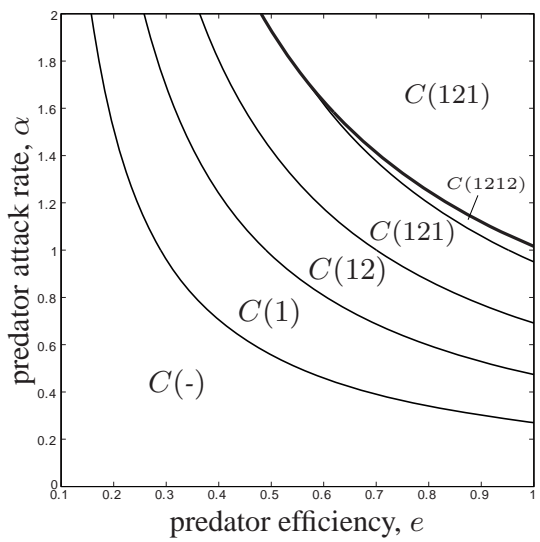

(e)

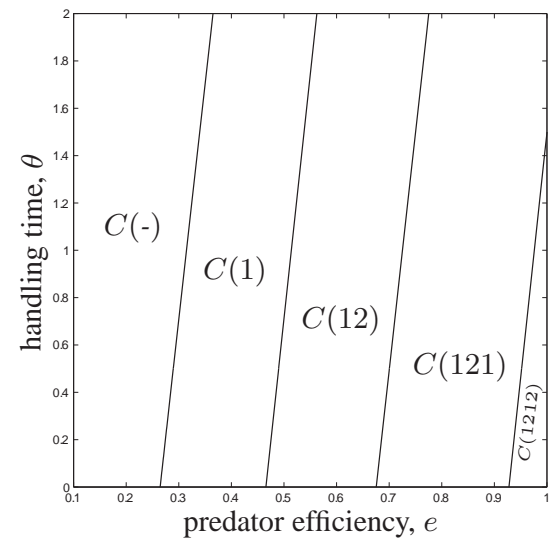

(b)

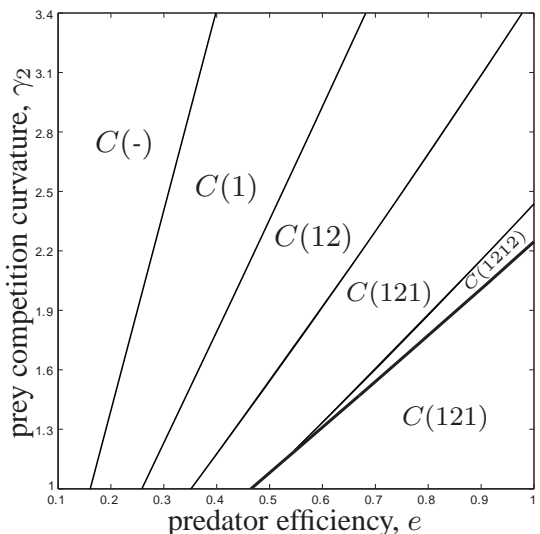

(d)

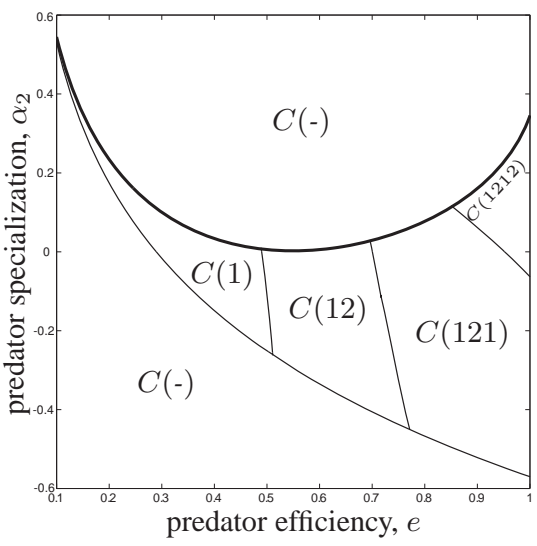

(f)

Fig. 6: Six examples of branching portraits obtained from Fig. 3 through continuation. Parameter values as in Fig. 3 and $\gamma_{3}=2$. Thick line: Hopf bifurcation curve. 
alternate between harvesting the prey with smaller (resp. larger) trait and the intermediate prey. Technically, evolutionary cycling is due to a Hopf bifurcation in the system at node $(3,2)$ (the thick curve in the figure) through which the evolutionary equilibrium becomes unstable and surrounded by a stable limit cycle. Recall that evolutionary cycling prevents further branching, as branching requires evolution to settle down at an evolutionary equilibrium, so the sequence $s=121$ is complete below the Hopf curve (see the lower set $C(121)$ ).

Also the influence of the predator maximum attack rate is relevant (Fig. 6(e)). Increasing the predation pressure favors prey branching, which in turn makes selection disruptive on the predator. Again increasing the attack rate destabilizes the evolutionary dynamics and prevents further branching (see the Hopf bifurcation in the system at node $(3,2)$ ). Red Queen evolutionary cycles of the kind just described for Fig. 6(d) develop in the upper set $C(121)$.

Finally, Fig. 6(f) shows that a predator with given efficiency promotes alternate branchings only if the kurtosis of the attack rate has intermediate values. If the kurtosis is decreased, i.e., if predator become more generalist, branching sequences become shorter until no branching is possible anymore (lower set $C(-)$ ). In contrast, if kurtosis increases, i.e., if predator become more specialist, longer branching sequences are first promoted but then made impossible by a Hopf bifurcation involving the monomorphic equilibrium of the ancestral community at the root node $(1,1)$ (see the upper set $C(-)$ ). As far as we know, this latter property was not discovered until now, while the former is in agreement with the literature (see [32, 50]).

We close this discussion by describing how a general method for investigating branching scenarios in two (or more) coevolving species could be derived from our analysis. First of all, evolutionary extinction should be considered by allowing sequences of four (instead of two) different events, namely branching and extinction of one or the other species, and extinctions events would be represented by backwards links in the graph of Fig. 2, which would no longer be a tree. This would allow the study of interesting systems, e.g. the evolution of cannibalism [12], where the existence of branching-extinction cycles has already been established [6]. Technically, evolutionary extinctions can be detected during continuation and simulation of the slow-fast system (15) as the collision of evolutionary trajectories with bifurcation boundaries of the ecological system (the fast compartment). Two types of bifurcations are responsible of evolutionary extinctions: the saddle-node bifurcation at which the ecological equilibrium (the node) collides with a saddle equilibrium and disappear; the transcritical bifurcation at which the density of a population vanishes. In the first case, the coevolution drives the community toward the loss of the ecological equilibrium of coexistence, after which the ecological system switches (on the fast ecological timescale) to another attractor, typically to another equilibrium at which some (or even all) populations are no longer present (evolutionary suicide [30,13]). In the second case, the density of a population is driven to zero by the evolution of the others (evolutionary murder [13]).

Another aspect not highlighted by our application is the possibility that the fitness derivative $B_{i}^{\prime \prime}$ changes sign while moving the model parameters. The points in parameter space where this occurs can be detected during continuation (see Appendix for the details on the computation of $B_{i}^{\prime \prime}$ ) and the curves along which $B_{i}^{\prime \prime}=0$ accordingly traced.

Worth to be mentioned is the choice of the ancestral condition of interest. The ancestral community for a case with two species should not necessarily be the $(1,1)$-community. Interesting cases where no branching is possible in the simplest community, whereas long branching sequences are observable starting from richer communities have indeed been discussed [37].

Moreover, rich and interesting branching scenarios for the selected ancestral community can be observable starting from some initial conditions and not starting from others. Multiple attractors in the fast (ecological) and in the slow (evolutionary) compartments can in fact be 
present $[8,11]$. Keeping track of all possible observable branching sequences is possible, but the description of a method greatly simplifies if one focuses on a specific initial condition.

Of course the bifurcations involving the selected ecological and evolutionary equilibria may force the community to switch (on the ecological or evolutionary timescale) to other attractors. In our analysis we always had coexistence at stable ecological equilibria (numerically checked), even though the AD canonical equation can be (heuristically) generalized to the case of nonstationary ecological attractors $[16,10]$.

But attractor switchings can also occur without involving bifurcations. In fact, in cases with multiple evolutionary attractors, the boundaries of the various basins of attraction (which are the stable manifold of saddle equilibria) do change along with the model parameters, so that the selected ancestral condition, or the initial condition imposed by a branching point on the next canonical equation, can pass from one basin of attraction to another at a critical parameter combination. Technically, this is not a bifurcation, but involves a qualitative change in the evolutionary dynamics, and therefore in the branching scenario produced from the initial condition under consideration. In some cases, the boundaries of the attraction basins can be continued, as suitable heteroclinic trajectories connecting saddle equilibria [40, 47], but most often basins of attraction are estimated by means of systematic simulations.

Keeping track of all the above possibilities in the formal description of a general method of analysis is quite involved and not among the aims of this paper. In our analysis we have emphasized only the aspects that were relevant for the considered application. Of course, of each computed curve (along which a branching condition vanishes, a bifurcation of the slow-fast system occurs, or the ancestral condition changes basin of attraction), only the segments for which the corresponding evolutionary equilibrium is reached from the considered ancestral condition matter in the branching portrait. Such segments are part of the branching portrait only if the change produced in the evolutionary dynamics induces a change in the branching sequence. This can be noticed, e.g., in Fig. 6(d,e), where the curve $B_{4,5}^{\prime}=0$ separating regions $C(121)$ and $C(1212)$ (on which the two predators acquire branching) merges with the Hopf bifurcation of the slow-fast system corresponding to node $(3,2)$. Moving rightto-left, the curve $B_{4,5}^{\prime}=0$ is part of the branching portrait only up to the contact with the Hopf curve.

Concluding remarks. We have analyzed the evolution of biodiversity in a prey-predator coevolutionary model based on the standard Rosenzweig-MacArthur ecological model [51]. Neglecting the introduction of alien species and accidental or artificial extinctions, we consider evolutionary branching $[27,25,13]$ - the coexistence and further differentiation of resident-mutant phenotypes - and evolutionary extinction [30, 13] — evolution toward selfor other-distruction-as the major drivers of biodiversity. Adaptive dynamics (AD [49, 27, $25,13,24]$ ) is the most suited modeling approach to investigate evolutionary branching and extinction in coevolutionary models, and the $\mathrm{AD}$ canonical equation makes it possible on a deterministic ground $[16,13]$.

We opted for measuring biodiversity with the number of phenotypically different prey and predator populations, thus losing information on the actual phenotypic values, but acquiring simplicity and compactness of representation. In particular, we have not found extinction events in the analyzed model, so that biodiversity evolves according to branching sequences, namely sequences of symbols 1 and 2 identifying branching in the prey and predator species, respectively.

We have discovered that long alternate (prey and predator) as well as unilateral (prey) branching sequences can occur. But we have also discovered that long sequences composed of a first phase of alternate branching concatenated with a long unilateral sequence of prey branchings are possible in some regions of parameter space. This explains why prey popula- 
tions can be much more numerous than predator populations, a fact that is often mentioned in field studies and in agreement with traditional [31, 42, 43, 41] and modern [18, 19, 29, 48] theories of competitive exclusion.

Another interesting result is that branching sequences become longer if predator become more and more specialist, until a critical point is reached at which never ending Red Queen ups and downs of the coevolving traits prevent a halt at evolutionary equilibria and therefore evolutionary branching. This discontinuity occurs at the birth of a stable evolutionary cycle due to a Hopf bifurcation of the evolutionary system.

Finally, critical parameter combinations for which branching scenarios are highly sensitive to parameter perturbations have been identified. This knowledge is of strategic importance for the conservation and management of biodiversity.

Our iterative procedure is based on simulations of ODEs (the AD canonical equation and the underlying ecological models) and continuations of algebraic systems of equations [1] and explores, at each iteration, the nature of longer and longer branching sequences. At each iteration, a better approximation of a two-dimensional branching portrait (explaining the dependence of branching scenarios on two parameters) becomes available. Our approach is more interesting, both computationally and conceptually, than the stochastic individualor population-based simulations mainly used until now when analytical tractability is unfeasible. Each simulation, even the deterministic one shown in Fig. 5, can only reveal an observable branching sequence, whereas our systematic analysis extracts information on all possible sequences including their nature, whether complete, incomplete or not observable. A particularly attractive feature is that, after a first branching portrait has been produced, the dependence of the branching scenarios on other parameters can be discussed without significantly increase the computational burden.

In principle, our approach can be made more general in view of investigating branching and extinction scenarios in AD models with two (or more) coevolving species, e.g., different prey-predator and host-parasite communities (see, e.g., [2]), as well as communities regulated by other ecological interactions (e.g., mutualistic [22] and competitive [37] communities). Although the formulation of an iterative algorithm is basically impossible-exactly for the same reasons why bifurcation analysis cannot be made fully automatic [40, 47]—we have discussed, partly in light of the specific model we have analyzed, the guidelines of a general method. In particular, evolutionary extinctions could be taken into account by considering sequences of different events, identifying branching and extinction in each of the coevolving species.

The indications that can be obtained by the proposed approach typically have qualitative nature, as those we have drawn for prey-predator coevolution, and should be checked not to be too specific for the considered model. However, dealing with rather complex long-term dynamics, we believe that this type of analysis can be of great help and should be considered for the long-term conservation and management of biodiversity.

Acknowledgments. The authors are grateful to Ulf Dieckmann who invited them to join the EEP group at IIASA during Summer 2011 and 2012 and suggested the analysis of the impact of the kurtosis of the predator attack rate on branching scenarios. The contribution of the anonymous reviewers is also acknowledged. The study has been supported by Ministero dell'Istruzione, dell'Università e della Ricerca, under project FIRB Futuro in Ricerca "Modeling and Analysis of Innovation and Competition Processes", contract RBFR08TIA4.

Appendix A. Computation of the fitness derivative $B_{i}^{\prime \prime}$. We show in this appendix how the branching condition (7) can be numerically computed, with reference to the simplest community composed of $N=2$ populations. Generalization to the case with $N>2$ is straightforward. 
The invasion fitness $\lambda_{i}$ is a function of the resident and mutant traits $\left(x_{1}, x_{2}, x_{i}^{\prime}\right), i=1,2$, that is obtained by evaluating the mutant per-capita growth rate (the initial exponential rate of increase of the mutant population) at the resident equilibrium of model (1), i.e.,

$$
\lambda_{i}\left(x_{1}, x_{2}, x_{i}^{\prime}\right)=f_{i}^{\prime}\left(\bar{n}_{1}\left(x_{1}, x_{2}\right), \bar{n}_{2}\left(x_{1}, x_{2}\right), 0, x_{1}, x_{2}, x_{i}^{\prime}\right) .
$$

When taking the fitness derivative with respect to $x_{j}, j=1,2$, one obtains

$$
\begin{aligned}
\frac{\partial}{\partial x_{j}} \lambda_{i}\left(x_{1}, x_{2}, x_{i}^{\prime}\right)= & {\left[\frac{\partial}{\partial n_{1}} f_{i}^{\prime}\left(n_{1}, n_{2}, 0, x_{1}, x_{2}, x_{i}^{\prime}\right) \frac{\partial}{\partial x_{j}} \bar{n}_{1}\left(x_{1}, x_{2}\right)+\right.} \\
& \frac{\partial}{\partial n_{2}} f_{i}^{\prime}\left(n_{1}, n_{2}, 0, x_{1}, x_{2}, x_{i}^{\prime}\right) \frac{\partial}{\partial x_{j}} \bar{n}_{2}\left(x_{1}, x_{2}\right)+ \\
& \left.\frac{\partial}{\partial x_{j}} f_{i}^{\prime}\left(n_{1}, n_{2}, 0, x_{1}, x_{2}, x_{i}^{\prime}\right)\right]\left.\right|_{n_{1,2}=\bar{n}_{1,2}\left(x_{1}, x_{2}\right)},
\end{aligned}
$$

where the explicit expressions for the derivatives of the resident equilibrium with respect to the traits are typically not available.

They can however be computed recalling the definition of $\bar{n}(x)$, i.e.,

$$
\begin{aligned}
& F_{1}\left(\bar{n}_{1}\left(x_{1}, x_{2}\right), \bar{n}_{2}\left(x_{1}, x_{2}\right), x_{1}, x_{2}\right)=0 \\
& F_{2}\left(\bar{n}_{1}\left(x_{1}, x_{2}\right), \bar{n}_{2}\left(x_{1}, x_{2}\right), x_{1}, x_{2}\right)=0
\end{aligned}
$$

(see model (1)). In fact, taking the derivative of (A1) with respect to $x_{j}$ gives

$$
\begin{aligned}
& \frac{\partial F_{1}}{\partial n_{1}} \frac{\partial \bar{n}_{1}}{\partial x_{j}}+\frac{\partial F_{1}}{\partial n_{2}} \frac{\partial \bar{n}_{2}}{\partial x_{j}}+\frac{\partial F_{1}}{\partial x_{j}}=0 \\
& \frac{\partial F_{2}}{\partial n_{1}} \frac{\partial \bar{n}_{1}}{\partial x_{j}}+\frac{\partial F_{2}}{\partial n_{2}} \frac{\partial \bar{n}_{2}}{\partial x_{j}}+\frac{\partial F_{2}}{\partial x_{j}}=0
\end{aligned}
$$

where the functions' arguments have been omitted for simplicity, but note that $\partial F_{1,2} / \partial x_{j}=$ $\partial F_{1,2}\left(n_{1}, n_{2}, x_{1}, x_{2}\right) /\left.\partial x_{j}\right|_{n_{1,2}=\bar{n}_{1,2}\left(x_{1}, x_{2}\right)}$.

The equations in (A2) form a linear system in the unknowns $\partial \bar{n}_{1} / \partial x_{j}$ and $\partial \bar{n}_{2} / \partial x_{j}$. In matrix form, it is

$$
J\left[\begin{array}{l}
\frac{\partial \bar{n}_{1}}{\partial x_{j}} \\
\frac{\partial \bar{n}_{2}}{\partial x_{j}}
\end{array}\right]=-\left[\begin{array}{l}
\frac{\partial F_{1}}{\partial x_{j}} \\
\frac{\partial F_{2}}{\partial x_{j}}
\end{array}\right],
$$

where $J$ is the Jacobian matrix associated with the resident equilibrium, that is hyperbolic (and therefore invertible) by assumption. 


\section{REFERENCES}

[1] E. L. Allgower And K. Georg, Introduction to Numerical Continuation Methods, SIAM Classics in Applied Mathematics, 2003.

[2] A. Best, A. White, E. Kisdi, J. Antonovics, M. A. Brockhurst, And M. Boots, The evolution of host-parasite range, The American Naturalist, 176 (2010), pp. 63-71.

[3] F. BRiand And J. E. Cohen, Community food webs have scale-invariant structure, Nature, 307 (1984), PP. 264-267.

[4] N. Champagnat, R. Ferrière, And S. MÉLÉARD, Unifying evolutionary dynamics: From individual stochastic processes to macroscopic models, Theoret. Population Biology, 69 (2006), pp. 297-321.

[5] D. L. De Angelis And L. J. Gross, eds., Individual-Based Models and Approaches in Ecology: Populations, Communities and Ecosystems, Chapman \& Hall, New York, 1992.

[6] F. DeRCOLE, Remarks on branching-extinction evolutionary cycles, J. Math. Biol., 47 (2003), pp. 569-580.

[7] F. Dercole, U. Dieckmann, M. Obersteiner, and S. Rinaldi, Adaptive dynamics and technological change, Technovation, 28 (2008), pp. 335-348.

[8] F. DERCOLE, R. FERRIÈRE, AND S. RINALDI, Ecological bistability and evolutionary reversals under asymmetrical competition, Evolution, 56 (2002), pp. 1081-1090.

[9] - Chaotic Red Queen coevolution in three-species food chains, Proc. R. Soc. Lond. B, 277 (2010), pp. 2321-2330.

[10] F. Dercole, A. Gragnani, R. Ferrière, And S. Rinaldi, Coevolution of slow-fast populations: evolutionary sliding, evolutionary pseudo-equilibria and complex Red Queen dynamics, Proc. R. Soc. Lond. B, 273 (2006), pp. 983-990.

[11] F. DERCOle, J. O. IRISSON, AND S. RinAldi, Bifurcation analysis of a prey-predator coevolution model, SIAM J. Appl. Math., 63 (2003), pp. 1378-1391.

[12] F. Dercole And S. Rinaldi, Evolution of cannibalistic traits: Scenarios derived from adaptive dynamics, Theoret. Population Biology, 62 (2002), pp. 365-374.

[13] - Analysis of Evolutionary Processes: The Adaptive Dynamics Approach and Its Applications, Princeton University Press, 2008.

[14] - Evolutionary dynamics can be chaotic: A first example, Internat. J. Bifur. Chaos, 11 (2010), pp. 34733485.

[15] U. Dieckmann And M. Doebeli, On the origin of species by sympatric speciation, Nature, 400 (1999), pp. 354-357.

[16] U. DiEcKmann AND R. LAW, The dynamical theory of coevolution: A derivation from stochastic ecological processes, J. Math. Biol., 34 (1996), pp. 579-612.

[17] U. Dieckmann, U. Marrow, And R. LAw, Evolutionary cycling in predator-prey interactions: Population dynamics and the Red Queen, J. Theoret. Biol., 176 (1995), pp. 91-102.

[18] O. Diekmann, M. Gyllenberg, and J. A. J. Metz, Steady state analysis of structured population models, Theoret. Population Biology, 63 (2003), pp. 309-338.

[19] U. Diekmann And J. A. J. Metz, Surprising evolutionary predictions from enhanced ecological realism, Theoret. Population Biology, 69 (2006), pp. 263-281.

[20] M. Doebeli And U. Dieckmann, Evolutionary branching and sympatric speciation caused by different types of ecological interactions, The American Naturalist, 156 (2000), pp. 77-101.

[21] M. Durinx, J. A. J. Metz, And G. MeszénA, Adaptive dynamics for physiologically structured population models, J. Math. Biol., 56 (2008), pp. 673-742.

[22] R. Ferrière, J. L. Bronstein, S. Rinaldi, R. Law, and M. Gauduchon, Cheating and the evolutionary stability of mutualisms, Proc. R. Soc. Lond. B, 269 (2002), pp. 773-780.

[23] S. A. H. Geritz, Resident-invader dynamics and the coexistence of similar strategies, J. Math. Biol., 50 (2005), pp. 67-82

[24] S. A. H. Geritz And F. Dercole, Editorial, J. Biol. Dyn., 5 (2011), p. 103.

[25] S. A. H. Geritz, E. Kisdi, G. MeszénA, And J. A. J. Metz, Evolutionarily singular strategies and the adaptive growth and branching of the evolutionary tree, Evolutionary Ecology, 12 (1998), pp. 35-57.

[26] S. A. H. GERITZ, E. Kisdi, AND P. YAn, Evolutionary branching and long-term coexistence of cycling predators: Critical function analysis, Theoret. Population Biology, 71 (2007), pp. 424-435.

[27] S. A. H. Geritz, J. A. J. Metz, E. Kisdi, And G. MeszénA, The dynamics of adaptation and evolutionary branching, Phys. Rev. Lett., 78 (1997), pp. 2024-2027.

[28] S. A. H. Geritz, E. VAn Der Meijden, And J. A. J. Metz, Evolutionary dynamics of seed size and seedling competitive ability, Theoret. Population Biology, 55 (1999), pp. 324-343.

[29] M. Gyllenberg And G. Meszéna, On the impossibility of coexistence of infinitely many strategies, J. Math. Biol., 50 (2005), pp. 133-160.

[30] M. Gyllenberg And K. Parvinen, Necessary and sufficient conditions for evolutionary suicide, Bulletin of Mathematical Biology, 63 (2001), pp. 981-993.

[31] G. Hardin, The competitive exclusion principle, Science, 131 (1960), pp. 1292-1298. 
[32] E. Hernández-García, C. López, S. Pigolotti, And K. H. Andersen, Species competition: coexistece, exclusion and clustering, Philos. Trans. R. Soc. Lond. A, 367 (2009), pp. 3183-3195.

[33] S. Hsu, S. HubBel, And P. WAltmann, Competing predators, SIAM J. Appl. Math., 35 (1978), pp. 617625.

[34] G. E. Hutchinson, Homage to Santa Rosalia or why are there so many kinds of animals?, The American Naturalist, 93 (1959), pp. 145-159.

[35] I. C. ITO AND T. IKegAmi, Food-web formation with recursive evolutionary branching, J. Theoret. Biol., 238 (2006), pp. 1-10.

[36] A. A. King AND W. M. Schaffer, The geometry of a population cycle: A mechanistic model of snowshoe hare demography, Ecology, 3 (2001), pp. 814-830.

[37] E. KISDI, Evolutionary branching under asymmetric competition, J. Theoret. Biol., 197 (1999), pp. 149-162.

[38] E. KisDi AND S. A. H. GERITZ, Adaptive dynamics in allele space: Evolution of genetic polymorphism by small mutations in a heterogeneous environment, Evolution, 53 (1999), pp. 993-1008.

[39] E. Kisdi, F. J. A. JACOBS, AND S. A. H. GERITZ, Red Queen evolution by cycles of evolutionary branching and extinction, Selection, 2 (2001), pp. 161-176.

[40] Yu. A. Kuznetsov, Elements of Applied Bifurcation Theory, Springer Verlag, 3rd ed., 2004.

[41] R. H. MacArthur, Species packing, and what interspecies competition minimizes, Proc. Natl. Acad. Sci., 64 (1969), pp. 1369-1371.

[42] R. H. MACARTHUR AND R. LEVINS, Competition, habitat selection and character displacement in a patchy environment, Proc. Natl. Acad. Sci., 51 (1964), pp. 1207-1210.

[43] - The limiting similarity, convergence and divergence of coexisting species, The American Naturalist, 101 (1967), pp. 377-385.

[44] P. Marrow, U. Dieckmann, AND R. LAW, Evolutionary dynamics of predator-prey systems: An ecological perspective, J. Math. Biol., 34 (1996), pp. 556-578.

[45] P. Marrow, R. LAW, AND C. CAnNings, The coevolution of predator-prey interactions: ESSs and Red Queen dynamics, Proc. R. Soc. Lond. B, 250 (1992), pp. 133-141.

[46] N. D. MARTINEZ, Network evolution: exploring the change and adaptation of complex ecological systems over deep time, in Ecological Networks: Linking Structure to Dynamics in Food Webs, Oxford University Press, 2006, pp. 287-301.

[47] H. G. E. Meijer, F. Dercole, and B. E. Oldeman, Numerical bifurcation analysis, in Encyclopedia of Complexity and System Science, R. A. Meyers, ed., 2009, pp. 6329-6352.

[48] G. Meszéna, M. Gyllenberg, L. PÁsztor, And J. A. J. Metz, Competitive exclusion and limiting similarity: A unified theory, Theoret. Population Biology, 69 (2006), pp. 68-87.

[49] J. A. J. Metz, S. A. H. Geritz, G. Meszéna, F. J. A. Jacobs, And J. S. Van HeerwaArden, Adaptive dynamics: A geometrical study of the consequences of nearly faithful reproduction, in Stochastic and Spatial Structures of Dynamical Systems, S. J. van Strien and S. M. Verduyn Lunel, eds., Elsevier Science, 1996, pp. 183-231.

[50] S. Pigolotti, C. López, E. Hernández-García, And K. H. Andersen, How gaussian competition leads to lumpy or uniform species distributions, Theoretical Ecology, 3 (2010), pp. 89-96.

[51] M. L. Rosenzweig And R. H. MacArthur, Graphical representation and stability conditions of predator-prey interactions, The American Naturalist, 97 (1963), pp. 209-223.

[52] A. SASAKI, Clumped distribution by neighborhood competition, J. Theoret. Biol., 186 (1997), pp. $415-430$.

[53] M. Scheffer, Ecology of Shallow Lakes, Kluwer Academic, 1st ed., 1998.

[54] M. SCHEFFER AND E. H. VAN NES, Self-organized similarity, the evolutionary emergence of groups of similar species, Proc. Natl. Acad. Sci., 16 (2006), pp. 6230-6235.

[55] P. Turchin, Complex Population Dynamics: A Theoretical/Empirical Synthesis, Princeton University Press, 2003.

[56] L. VAn Valen, A new evolutionary law, Evolutionary Theory, 1 (1973), pp. 1-30. 